\title{
Integrated optical devices using bacteriorhodopsin as active nonlinear optical material
}

\author{
András Dér* ${ }^{\mathrm{a}}$, László Fábián ${ }^{\mathrm{a}}$, Sándor Valkai ${ }^{\mathrm{a}}$, Elmar Wolff ${ }^{\mathrm{b}}$, Jeremy Ramsden ${ }^{\mathrm{c}}$ and Pál Ormos ${ }^{\mathrm{a}}$ \\ ${ }^{a}$ Institute of Biophysics, Biological Research Center of the Hungarian Academy of Sciences, H- \\ 6701 Szeged, P.O.B. 521 \\ ${ }^{\mathrm{b}}$ Institute for Applied Biotechnology and System Analysis, University of Witten-Herdecke, D- \\ 58455 Witten \\ ${ }^{\mathrm{c}}$ School of Industrial and Manufacturing Science, Cranfield University, Cranfield, Badfordshire \\ MK43 0AL, England
}

\begin{abstract}
Coupling of optical data-processing devices with microelectronics, telecocommunication and sensory functions, is among the biggest challenges in molecular electronics. Intensive research is going on to find suitable nonlinear optical materials that could meet the demanding requirements of optoelectronic applications, especially regarding high sensitivity and stability. In addition to inorganic and organic crystals, biological molecules have also been considered for use in integrated optics, among which the bacterial chromoprotein, bacteriorhodopsin (bR) generated the most interest. bR undergoes enormous absorption and concomitant refractive index changes upon initiation of a cyclic series of photoreactions by a burst of actinic light. This effect can be exploited to create highly versatile all-optical logical elements. We demonstrate the potential of this approach by investigating the static and dynamic response of several basic elements of integrated optical devices. Our results show that, due to its relatively high refractive index changes, bR can be used as an active nonlinear optical material to produce a variety of integrated optical switching and modulation effects.
\end{abstract}

Keywords: integrated optics, all-optical switching, bacteriorhodopsin

\section{INTRODUCTION}

Bacteriorhodopsin (bR), isolated from the outer cell membrane of the bacterium Halobacterium salinarum [1], is the simplest known ion pump, and one of the best-characterized membrane proteins. Upon illumination it transports protons across the membrane, while undergoing a cyclic series of reactions with quasi-stable intermediate states (called the photocycle) [2]:

$$
\begin{aligned}
& \mathrm{BR}_{570} \rightarrow \mathrm{K}_{620} \rightarrow \mathrm{L}_{540} \rightarrow \mathrm{M}_{410} \rightarrow \mathrm{N}_{550} \rightarrow \mathrm{O}_{630} \rightarrow \mathrm{BR}_{570} \\
& \mathrm{BR}_{570} \rightarrow \mathrm{K}_{620} \rightarrow \mathrm{L}_{540} \rightarrow \mathrm{M}_{410} \rightarrow \mathrm{N}_{550} \rightarrow \mathrm{O}_{630} \rightarrow \mathrm{BR}_{570}
\end{aligned}
$$

The letters denote the ground and intermediate states, and the subscripts refer to the wavelengths/nm of their absorption maxima. The transition rates in dried bR films span from subpicoseconds to seconds. Gels and thin films containing oriented bR molecules [3,4] are extremely stable, maintaining their photoelectric activity for several years at the same level. Despite the large variety of bioelectronic applications utilizing the favourable optical and photoelectric properties of bR and its mutants produced by genetic engineering [5], no report has yet been published about its application in integrated optics (IO). IO is a new discipline of optoelectronics, which integrates various optomodules on a small substrate, in order to create analogues of integrated circuits [6]. Since the theory and measuring techniques for integrated optics are well established, the main limitations in developing new devices are of a technical nature, namely, in our case to find the proper NLO material for the particular application envisaged. The Kramers-Kronig relations imply changes in

*derandra@brc.hu; phone 3662 599-607; fax 3662 433-133

Linear and Nonlinear Optics of Organic Materials VI, edited by Robert A. Norwood, Proc. of SPIE Vol. 6331, 633119, (2006) · 0277-786X/06/\$15 - doi: 10.1117/12.686173 
the absorption spectra accompanying changes in the index of refraction. The light-induced change of refractive index of a bR-containing film was indeed demonstrated earlier [7,8], though not kinetically resolved. This opens the way to a highly interesting use of the NLO characteristics of bR namely for the modulation of the coupling of light into and out of IO devices controlled by the light-induced transitions of $b R$.

\section{MATERIALS AND METHODS}

Bacteriorhodopsin-containing purple membranes were suspended in distilled water and dry bR-films of good optical quality were produced by layering the suspension on a smooth surface. Integrated optical experiments were carried out using slab geometry waveguides (Microvacuum Ltd, Budapest), and Mach-Zehnder interferometers.

\subsection{Slab waveguide experiments}

The slab waveguide consisted of a thin $\mathrm{Si}(\mathrm{Ti}) \mathrm{O}_{2}$ layer (refractive index $\mathrm{n}_{\mathrm{F}} \approx 1.8$, thickness $\mathrm{d}_{\mathrm{F}} \approx 200 \mathrm{~nm}$ ) on a $16 \times 48 \mathrm{~mm}$ glass substrate (refractive index $n_{\mathrm{S}} \approx 1.5$ ) surface. Coupling of light into the waveguide was achieved through a grating formed in the waveguide (line density: $1 / \Lambda=2400 \mathrm{~mm}^{-1}$, length of the grating region: $1 \mathrm{~mm}$ ). In a thin waveguide, only the zeroth transverse electric (TE) and transverse magnetic (TM) light modes can propagate [9,10]. Since the guided modes have a significant evanescent component, coupling to or from an external beam is strongly influenced by the refractive index of the material above the grating area (the adlayer). Thus, a change of the index of refraction of $b R$ $\left(\Delta \mathrm{n}_{\mathrm{A}}\right)$ will modulate the angle $\alpha$ of incoupling.

We determined the changes of incoupling angle during the bR photocycle initiated by a pulse from an excimer laser pumped dye laser (Lambda Physik Lextra 100, Rhodamine 6G, $\lambda=580 \mathrm{~nm}, \mathrm{E}=10 \mathrm{~mJ} / \mathrm{pulse}$ ) or from a Nd:YAG laser (Continuum) equipped with a tunable optical parametric oscillator head ( $\lambda=410 \mathrm{~nm}, 10 \mathrm{~mJ}$ pulse energy).

The waveguide was placed on a rotational stage (Ealing Electro Optics Digital Positioning System), by which the coupling angle $\alpha$ could be controlled to an accuracy of $10^{-4} \mathrm{deg}$. The incoupled light was measured at the ends of the waveguide by a photomultiplier (Hamamatsu). The light intensity signal was amplified by a laboratory-built preamplifier and then recorded with a Le Croy 9310L transient digitizer. In order to improve the signal to noise ratio, the time resolution of the system was limited by low-pass filtering to $0.8 \mu \mathrm{s}$.

\subsection{Experiments with Mach-Zehnder interferometer}

The integrated Mach-Zehnder interferometer structure was constructed of polymerized NOA81 (Norland Product, Inc.) optical adhesive. First a $9 \mu \mathrm{m}$ thick layer of the NOA81 was spincoated onto a glass cover slip. The layer on the cover slip was then taken to the motorized stage of an inverse microscope (Axiovert200, Zeiss). The beam of a Kr-ion laser (Innova 304, Coherent, $407 \mathrm{~nm}, 10 \mathrm{~mW}$ ) was focused onto the layer by means of a 10X microscope objective. A computer-controlled shutter and the stepper motors of the microscope stage controlled the writing of a Mach-Zehnder structure into the resin film. After the writing process was completed the non-exposed part (which is not hardened and stuck to the cover slip glass) was removed by acetone/ethanol 3:1 mixture. The width of the hardened lines was $10 \mu \mathrm{m}$. The physical dimensions of the structure allowed incoupling the light directly from a single-mode optical fiber core (5 $\mu \mathrm{m}$ in diameter). For this reason, the optical fiber was placed and fixed close to the end face of the stripe, finally all parts were fixed to a regular microscope slide that was used as a base plate of the structures (Figure 1A-D).

A water suspension of $\mathrm{pm}(\mathrm{pH} \mathrm{6,} \mathrm{OD} \mathrm{35)} \mathrm{was} \mathrm{layered} \mathrm{on} \mathrm{both} \mathrm{arms} \mathrm{of} \mathrm{the} \mathrm{Mach-Zehnder} \mathrm{interferometer} \mathrm{and} \mathrm{dried} \mathrm{at} 25$ ${ }^{\circ} \mathrm{C}$ under a laminar flow for 3 hours. The relative humidity of the films was adjusted to be $50 \%$. The transfer function of the interferometer was measured under a microscope (see above). An optical fiber was fixed at the output of the interferometer in order to guide the transmitted red light to a photomultiplier (Hamamatsu), and its signal was recorded by a digital storage oscilloscope (Le Croy). The beam of an Ar-ion laser (Stabilite2016, Spectraphysics) was used to excite the $\mathrm{bR}(\lambda=488 \mathrm{~nm}, 100 \mathrm{~mW})$, while the measuring light was that of a diode laser $(\lambda=676 \mathrm{~nm}, 8 \mathrm{~mW})$. 

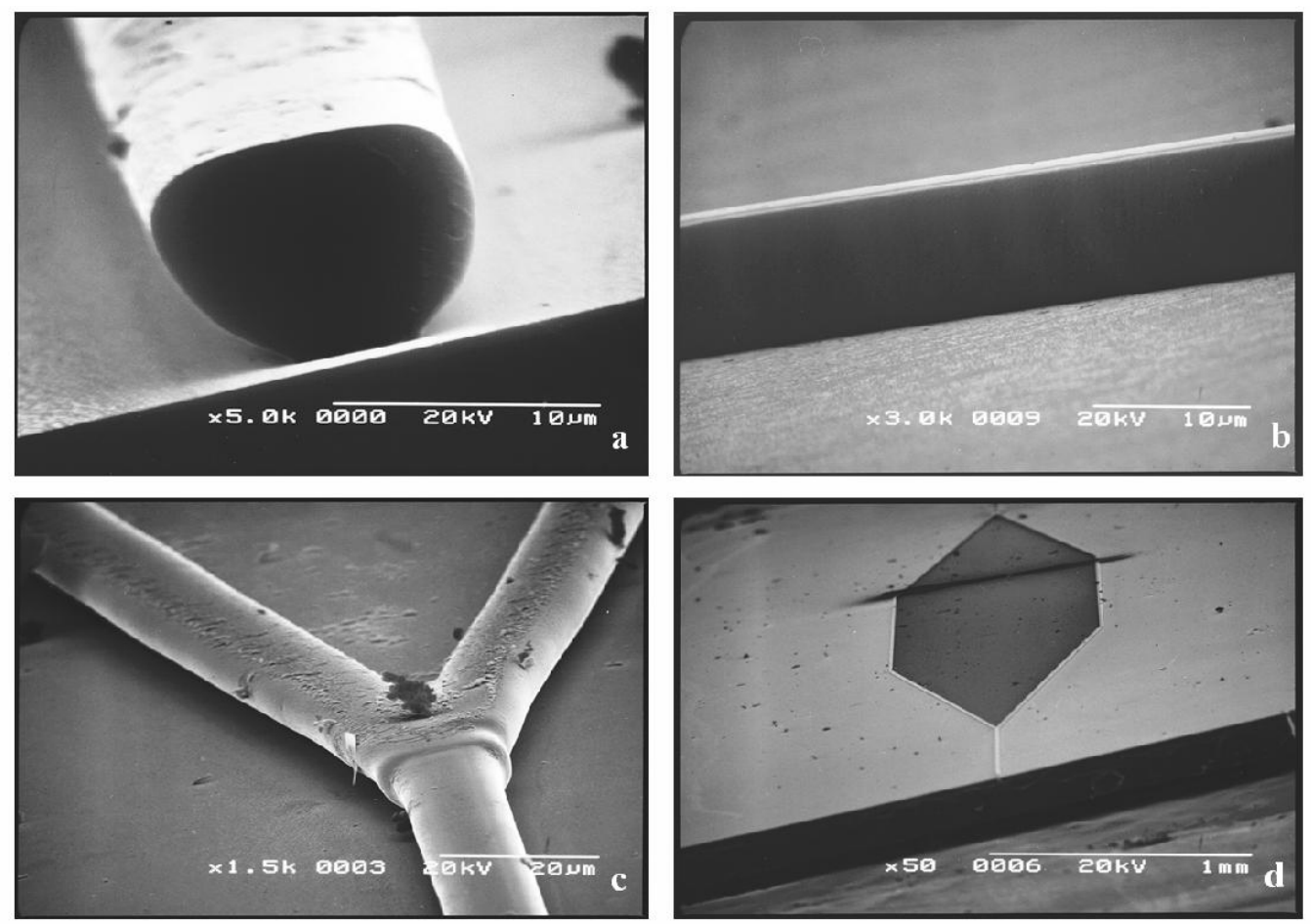

Fig. 1 A-D. Electron micrograph images of the photopolymeric Mach-Zehnder interferometer
A) Cross-sectional view of a single strip
B) Side view of a single strip
C) View of a Y-branch
D) View of the whole M-Z structure (shaded inside)

\section{RESULTS AND DISCUSSION}

\subsection{Slab waveguide experiments}

From the angular shift of the peaks of incoupling (compared to the case when no bR adlayer is present), the refractive index of the (unexcited) bR adlayer was determined to be to 1.52, in agreement with previous observations by other methods. ${ }^{9}{ }^{10}$ The rotational stage was then stopped at different angles in the region of effective coupling and the light intensity changes following flash excitation were measured.

Fig. 3. shows two traces measured at opposite sides of the incoupling peak corresponding to the TE mode. The traces were analyzed using global fitting with multiexponential curves. In the millisecond domain two exponentials resulted in a good fit, with time onstants $\tau_{1}=57 \mathrm{~ms}$ and $\tau_{2}=350 \mathrm{~ms}$, characteristic of the second half of the photocycle (M-decay) at low humidity [11]. 


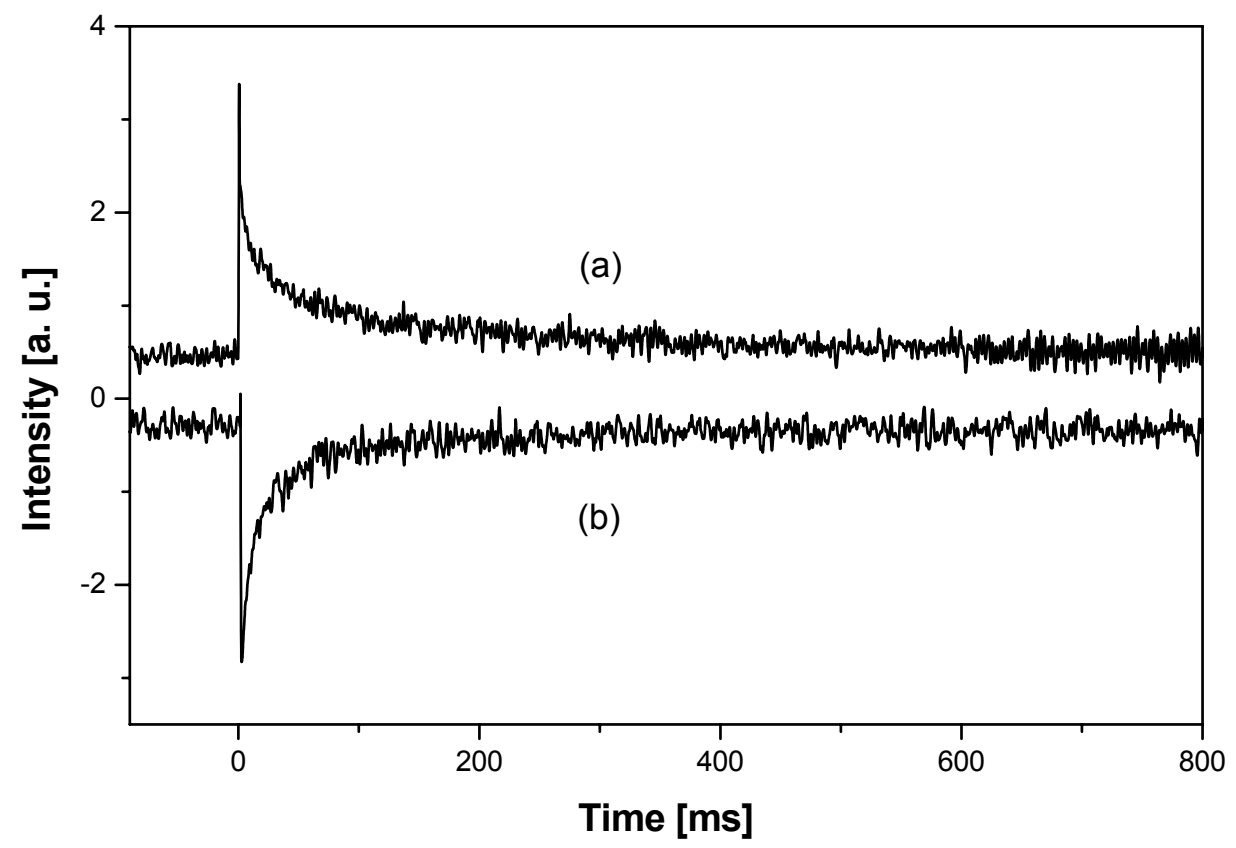

Fig. 2. Coupled light intensity changes measured upon flash excitation of the film. Ground state bR was excited by $570 \mathrm{~nm}$ flashes. The measuring light intensity was attenuated to $0.5 \mathrm{~mW}$ (He-Ne laser, $\lambda=633 \mathrm{~nm}$ ), so as to avoid considerable pre-excitation of the sample. Traces were recorded at two positions ( $\mathrm{a}$ and $\mathrm{b}$, with incoupling angles $\theta_{\mathrm{a}}=5.967^{\circ}$ and $\left.\theta_{\mathrm{b}}=6.167^{\circ}\right)$, on opposite sides of the incoupling peak at the transversal electric mode $\left(\theta_{\mathrm{TE}}=6.067^{\circ}\right)$. The amplitude of the relative light intensity change at $\theta_{\mathrm{a}}$ was about $70 \%$.

The antisymmetry of the traces show that the primary origin of the intensity change is a transient change of the coupling angle, confirming the dominating role of refractive index changes in the observed switching effect. From the maximal value of the angular shift, the corresponding light-induced refractive index change of the adlayer was calculated $\left(2 \times 10^{-3}\right)$ using the waveguide equations [10]. Its value is close to previous findings [7,8].

To be able to correlate the changes of the coupling geometry to the steps of the photocycle, we also performed absorption kinetic experiments on the same sample under identical conditions (not shown). Evaluating the kinetics of the $\mathrm{M}$ intermediate (obtained by measuring absorption changes of the bR film at $405 \mathrm{~nm}$ upon light excitation), we obtained the same time constants as mentioned above. The correlation is in agreement with the Kramers-Kronig origin of the observed refractive index changes on this time scale, corresponding to the nearly $160 \mathrm{~nm}$ difference between the absorption maxima of the bR ground and M states. The speed of light modulation based on this effect, therefore, should be limited by the kinetics of the $\mathrm{M}$ formation to about $50 \mu \mathrm{s}$ in the photocycle of wild type $\mathrm{bR}$, when using flash excitation [11]. Under continuous illumination, however, when the light density is much lower, the buildup of the M population $\left(\tau_{\mathrm{M}}\right)$, and consequently the refractive index changes are limited by the light intensity and the decay rate of $\mathrm{M}$, typically to the millisecond time).

These pioneering experiments revealed that a dry film containing native bacteriorhodopsin shows unique nonlinear optical properties (high refractive index change controllable by light of different colours) that are in many respects superior to those of the materials presently applied in integrated optics [12]. In the following, we demonstrate the first integrated optical application based on a miniature Mach-Zehnder interferometer performing a real switching effect by bacteriorhodopsin (efficiency higher than 90\%). 


\subsection{Experiments with Mach-Zehnder interferometer}

The principle of switching is based on a reversible change of the refractive index of the bacteriorhodopsin film on one of the arms of the interferometer. The interference of the light at the output can thereby be changed from constructive to destructive or the reverse. Without exposure, the optical environment and the effective refractive index stays the same in both arms. When illuminating only one of the covered arms, a variation in the index of refraction leads to a phase imbalance in the two arms, and interference at the output of the interferometer.

Figure 3 demonstrates the high efficiency of bR-based all-optical integrated optical switching. The exciting light intensity was adjusted to show the maximal effect $(15 \mathrm{~mW}, 90 \%$ intensity modulation, respectively). After switching on the exciting light the steady state is reached within a few seconds, while the decay reflects the relaxation of the refractive index of the bR film after the excitation was switched off.

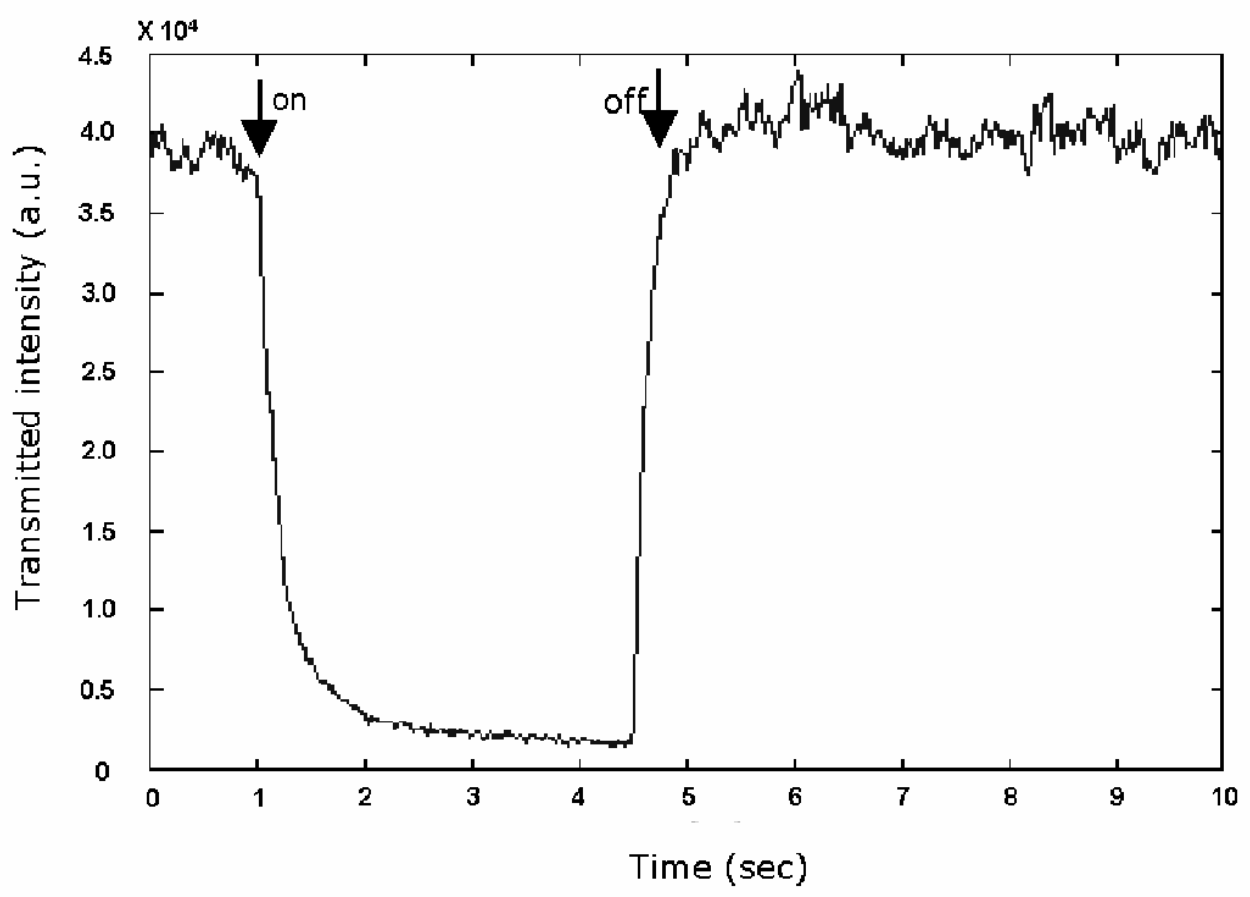

Fig. 3. Demonstration of the all-optical switching effect by the photopolymeric M-Z interferometer cover with a bR film as an adlayer. Guided light: diode laser, $670 \mathrm{~nm}$; Exciting light: 488nm Ar-ion laser.

Figure 4 shows the steady-state light intensity measured at the output of the Mach-Zehnder interferometer as a function of the exciting light intensity. In the investigated range, two minima and two maxima were observed, however, no saturation of the effect was found despite the high-intensity excitation. 


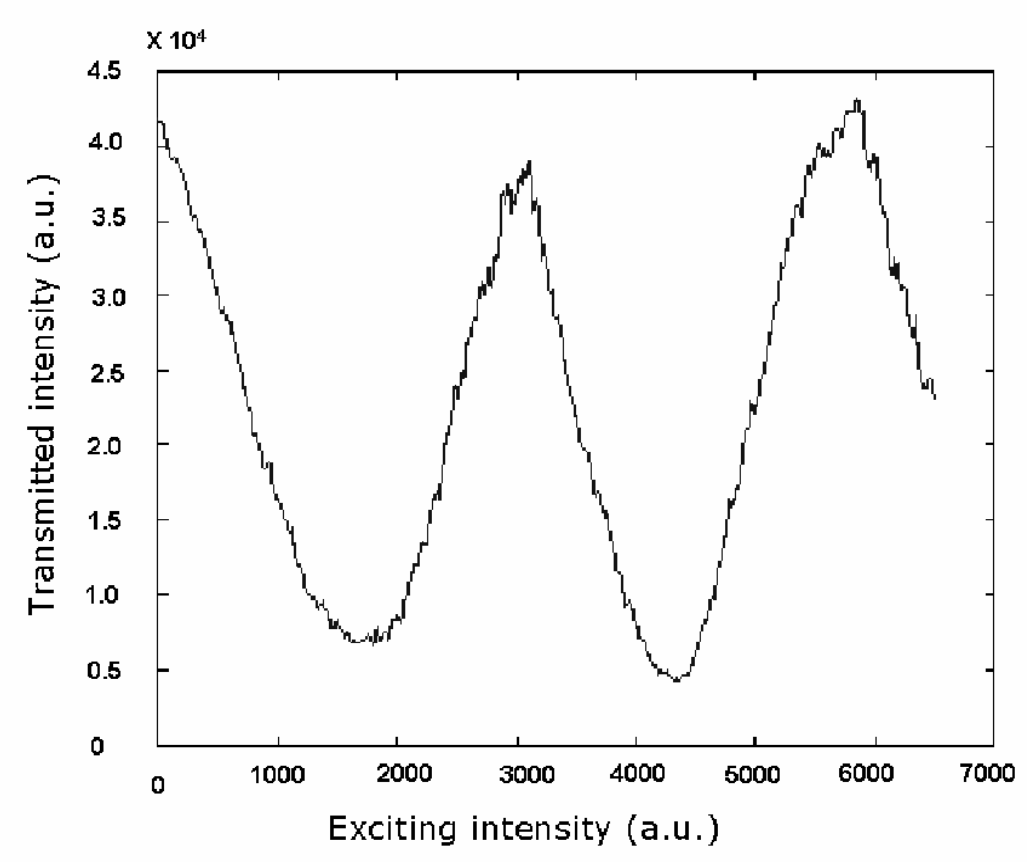

Fig. 4. Sinusoidal modulation of the transmitted red light as a function of exciting light intensity (sample as before).

In order to reveal the reasons for this unexpected finding, analogous experiments were performed on a single-striped waveguide structure. Here, contrary to the $\mathrm{M}-\mathrm{Z}$ structure (where the observed effects could be attributed to light-induced refractive index changes) only the absorption changes of the bR film were observed.
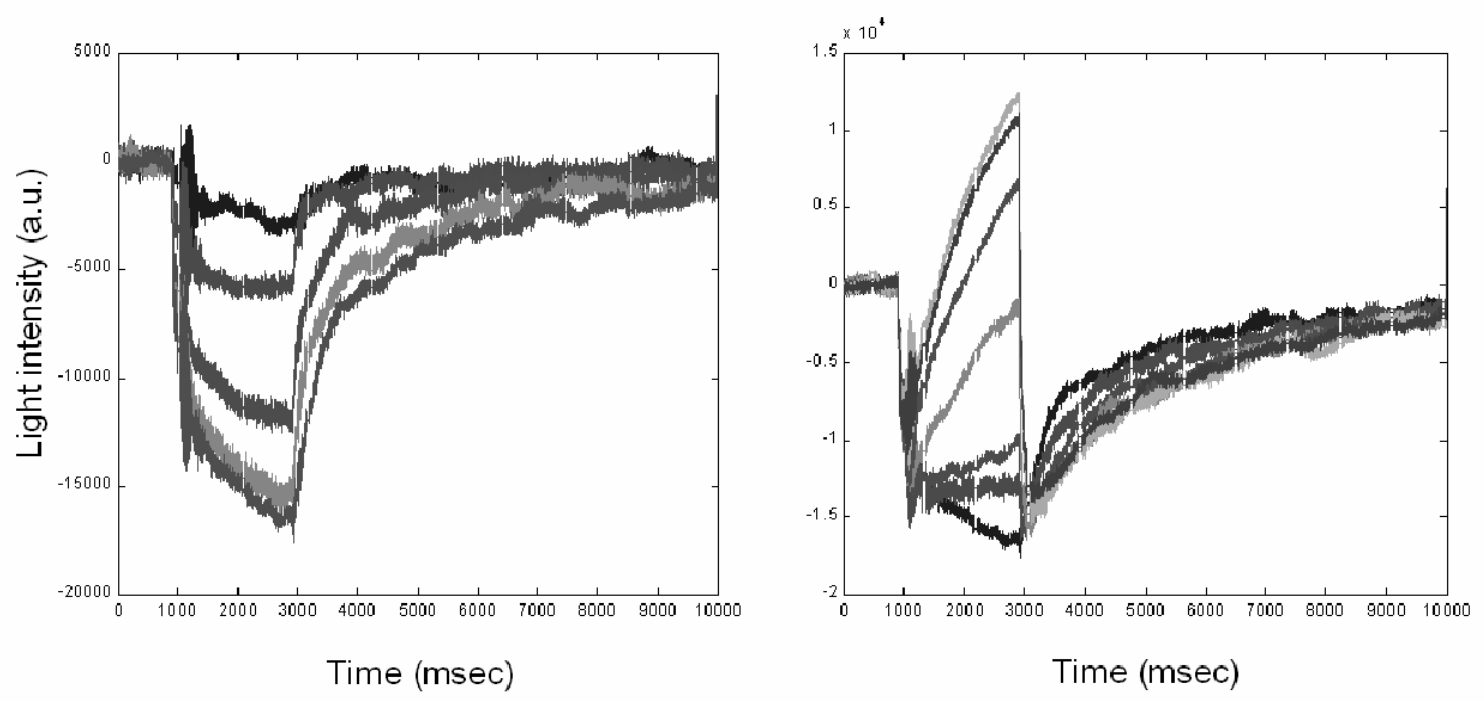

Fig.5. Changes in the output intensity of red measuring light guided by a single stripe covered with a bR film upon changing the intensity of exciting green light. Note that the signal increases first, then undergoes a turnover phase. 
Upon excitation by green light, due to its well-known photochromic effect, the protein-containing adlayer undergoes absorption changes giving rise to modification of the intensity of the measuring light. The effects are interpreted according to a simplified model describing the bR photocycle at moderate humidities, where the $\mathrm{N}$ and $\mathrm{O}$ forms are missing, and only the K, L and M intermediates are present (Figure 6).

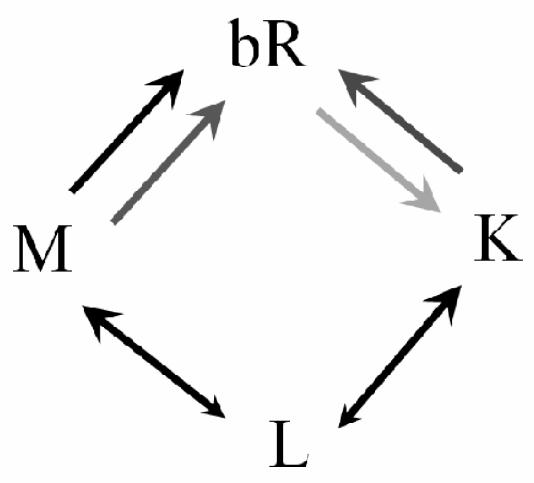

Fig. 6. A simplified scheme of the photocycle of dried bacteriorhodopsin

The red light intensity increase at lower levels of excitation (Fig. 5) is due to the accumulation of the $\mathrm{M}$ form in steadystate, where the longest-living intermediate is present with the highest weight. Under conditions where the concentration of $\mathrm{M}$ is maximal, the refractive index change of the film is $2 \times 10^{-4}$ as observed previously by the OWLS technique [12]. The subsequent absorption increase at $676 \mathrm{~nm}$ shows that another photoproduct is accumulated at higher light intensities, that is spectrally red-shifted compared to the ground-state bR. Note that after switching off the excitation, the absorption always returns to a minimum value corresponding to M-saturation before final relaxation. This finding strongly suggests that the red-shifted intermediate returns to the ground state (BR) via M. Taking into account the well-known fact that light excitation of both the $\mathrm{L}$ and $\mathrm{M}$ intermediates drives them back to the ground state [13], we suggest that the implicated red-shifted intermediate must be $\mathrm{K}$ or of its type.

In agreement with the experimental findings it is seen that while at low exciting light intensities $\mathrm{M}$ is accumulated, at higher values $\mathrm{K}$ dominates. At the highest applied light intensity the refractive index change of the film was estimated to be $-9 \times 10^{-4}$. This high value, which is comparable to that accompanying the BR-M transition but with negative sign, should be interpreted by the anomalous refractive index of $\mathrm{K}$ having an absorption maximum close to the wavelength of the measuring light.

These results suggest that besides $\mathrm{M}$, also the $\mathrm{K}$ intermediate has favorable non-linear optical properties (high refractive index change) that, due to the picosecond kinetics, may be readily be utilized in integrated optics or other nonlinear optical applications.

\section{CONCLUSIONS}

The above results demonstrate the feasibility of using a protein as a programmable active element in integrated optical devices. Hence the fabrication of microstructured all-optical devices coated with bR adlayer is primarily a technological task. The size, speed and cyclicity of the light-induced refractive index changes of $b R$ are comparable to or exceed the corresponding properties of inorganic NLO crystals, while the possibility of genetically controlling the optical properties of bR (absorption spectrum, cycle time, etc.) offers a great and unique versatility for optical circuit design.

\section{ACKNOWLEDGEMENTS}

This work was supported by a NATO SFP 974262 collaborative research grant. The authors are grateful to Drs. András Hámori and Kárpát Ferencz for providing waveguides used in preliminary experiments. 


\section{REFERENCES}

1. D. Oesterhelt and W. Stoeckenius, Methods Enzymol. 31, 667 (1974)

2. R. H. Lozier and W. Niederberger, Fed. Proc. 36, 1805 (1975)

3. G. Váró and L. Keszthelyi, Biophys. J. 43, 47 (1983)

4. A. Dér, P. Hargittai and J. Simon, J. Biochem. Biophys. Methods 10, 295 (1985)

5. R. R. Birge, Sci. Am. 272, 90-95 (1995)

6. K. Iizuka, in Engineering Optics, $2^{\text {nd }}$ edition, edited by Theodor Tamir (Springer-Verlag, London, 1987), Vol. 35, Chap. 15, p.408.

7. D. Zeisel and N. Hampp, J. Phys. Chem. 96, 7788 (1992).

8. C. P. Zhang, C. Y. Ku, Q. W. Song, R. B. Gross, and R. R. Birge, Opt. Lett. 19, 1409 (1994).

9. K. Tiefenthaler and W. Lukosz, J. Opt. Soc. Am. B 6, 209 (1989).

10. L. Guemori, J. Ogier, Z. Zekhnini, and J. J. Ramsden, J. Chem. Phys. 113, 8183 (2000).

11. G. Váró, In: Bioelectronic Applications of Photochromic Pigments, Szeged, Hungary, 149, (2000), edited by A. Dér IOS Press, Amsterdam, (2001).

12. P. Ormos, L. Fábián, L. Oroszi, E.K. Wolff, J.J. Ramsden and A. Dér, Appl. Phys. Lett. 80, 4060 (2002)

13. S. P. Balashov, Israel Journal of Chemistry 35, 415 (1995) 